\title{
Enzymatic depolymerization of highly crystalline polyethylene terephthalate enabled in moist-solid reaction mixtures
}

\author{
Sandra Kaabel, J. P. Daniel Therien, Catherine E. Deschênes, Dustin Duncan, Tomislav Friščić,* \\ Karine Auclair.* \\ Department of Chemistry \\ McGill University \\ 801 Sherbrooke St. W. H3A 0B8, Canada. \\ *Tomislav Friščić and Karine Auclair. \\ Email: tomislav.friscic@mcgill.ca or karine.auclair@mcgill.ca
}

Author Contributions: S.K. and J.P.D.T. conducted the research; D.D. contributed by the synthesis of the mono(2-hydroxyethyl) terephthalate standard; C.E.D. contributed with testing of the non-PET polymers; S.K. and J.P.D.T. wrote the original draft; K.A. and T.F. reviewed and edited the manuscript, in addition to supervising the research.

Competing Interest Statement: The authors report no competing interests.

Classification: Biological Sciences (Sustainability Science); Physical Sciences (Chemistry).

Keywords: PET hydrolysis, biocatalysis, mechanochemistry, cutinase, recycling.

\section{This PDF file includes:}

Main Text

Figures 1 to 4

\begin{abstract}
Less than $9 \%$ of the plastic produced is recycled after use, contributing to the global plastic pollution problem. While polyethylene terephthalate (PET) is one of the most common plastics, its thermomechanical recycling generates a material of lesser quality. Enzymes are highly selective, renewable catalysts active at mild temperatures; however, they lack activity towards the more crystalline forms of PET commonly found in consumer plastics, requiring the energy-expensive melt-amorphization step of PET before enzymatic depolymerization. We report here that when used in moist-solid reaction mixtures instead of the typical dilute aqueous solutions or slurries, the cutinase from Humicola insolens can directly depolymerize amorphous and crystalline regions of PET equally, without any pre-treatment, in 13-fold higher space-time yield and 15-fold higher enzyme efficiency than prior reports with high crystallinity material. Further, this process shows a 26-fold selectivity for terephthalic acid over other hydrolysis products.
\end{abstract}

\section{Introduction}

The amount of plastic manufactured annually is ever increasing, with 359 million tons produced in 2019.(1) Unfortunately, less than $9 \%$ of plastic waste is recycled, owing to the lack of economically feasible and sustainable recycling technologies.(2) With an estimated 70 million tons produced 
annually,(3) polyethylene terephthalate (PET) is one of the most common consumer plastics, making up disposable bottles and polyester clothing fibers, among others. Current recycling of PET, typically achieved through thermomechanical means, significantly reduces the polymer integrity,(4) thereby limiting the applications of recycled PET to lesser quality products that are eventually discarded (e.g. carpets).(5) Consequently, better PET recycling technologies are urgently needed to avoid the accumulation of plastic in landfills and the environment.(2)

Depolymerizing PET into terephthalic acid (TPA) and ethylene glycol (EG), allows for the production of high-quality PET products by re-polymerization.(6) Chemically breaking down PET, either though solvolysis or hydrolysis, typically involves the use of high temperatures $\left(>150^{\circ} \mathrm{C}\right)$ and/or pressures (up to $20 \mathrm{MPa}$ in supercritical conditions), catalysts, corrosive strong acids (e.g. sulfuric, nitric, or phosphoric acid) or bases $(e . g . \mathrm{NaOH}, \mathrm{KOH}),(7-11)$ producing large volumes of toxic waste and a TPA product often contaminated with glycol, alcohol and phthalate derivatives, incompatible with PET production.(12)

Biocatalysts capable of hydrolysing PET offer a more sustainable route to plastic recycling, as these environmentally benign, renewable catalysts can work under mild conditions and generate a clean product without using hazardous chemicals. Several enzymes capable of depolymerizing low crystallinity PET $(<20 \%)$ have been reported, including the remarkably efficient mutant of leafbranch compost cutinase, recently developed by Tournier et al:;(13) however, efficient depolymerization of highly crystalline forms (>20\%) of PET typically found in consumer products (Table S10) remains elusive.

Herein we show that using unconventional moist-solid reaction mixtures instead of standard aqueous solutions or slurries enables the direct enzymatic depolymerization of high crystallinity PET, avoiding thermal or chemical pre-treatment. When applied to the thermostable Humicola insolens cutinase (HiC, Novozym $\AA 51032)$, this unique approach yields TPA selectively, with simultaneous hydrolysis of both crystalline and amorphous PET, and with high purity suitable for direct up-cycling of the crude TPA. Overall, we show that enzymatic depolymerization of high crystallinity PET without bulk water proceeds with higher selectivity, enzyme efficiency, and with considerably improved space-time yield (STY) compared to prior reports (Table S10).

\section{Results and Discussion}

\section{Enzymatic hydrolysis of PET by HiC in moist solids}

Commercial PET powder of $36 \%$ crystallinity, similar to a post-consumer PET bottle, was used as a model substrate to develop our process (Figs. S2, S3). The reactions were typically carried out using a mechanoenzymatic approach,(14) based on an initial brief period of milling(15-17) PET in the presence of enzyme $(0.6 \mathrm{wt} \%)$ and a minimal amount of buffer (liquid-to-solid ratio $\eta=1.5 \mu \mathrm{L}$ $\mathrm{mg}^{-1}$, i.e. $667 \mathrm{mg} \mathrm{ml}^{-1}$ ), followed by 7 days of aging (static incubation) at $55^{\circ} \mathrm{C}$. (18-20) Notably, such reaction mixtures do not correspond to slurries or typical high-solids mixtures in which solvent effects are observed (as previously demonstrated for conditions of $\eta \geq 2$ ). ${ }^{14}$ Under moist-solid conditions, high crystallinity PET is depolymerized cleanly to TPA in $20 \pm 1 \%$ yield at a temperature well below the glass transition of the polymer (Fig. 1A-D, Table S1 entry 4). HPLC analysis of the crude mixture revealed 20 -fold selectivity for TPA over mono(2-hydroxyethyl) terephthalate (MHET) and no detectable amount of bis(2-hydroxyethyl) terephthalate (BHET) or other oligomers (Fig. 1D). This contrasts with an otherwise equivalent reaction performed under conventional aqueous conditions $\left(\eta=50 \mu \mathrm{L} \mathrm{mg}^{-1}\right.$, i.e. $\left.20 \mathrm{mg} \mathrm{ml}^{-1}\right)$ which proceeded in $10 \%$ yield and with only 2.8 -fold selectivity for TPA over MHET after 7 days (Table S1, entry 24). Remarkably, manual mixing of the reactants instead of ball milling was enough to obtain TPA in $19 \pm 1 \%$ yield within 7 days (Fig. $2 \mathrm{~A}$, Table S1 entry 1). To ensure reproducible mixing of the reactants, however, ball milling was used in all subsequent experiments. Attempt to conduct the reaction under identical conditions, but in the absence of enzyme, led to only $0.02 \%$ conversion to TPA (Table S1 entry 25).

The optimal aging temperature was found to be $45-55^{\circ} \mathrm{C}$ (Fig. 2B, Table S1 entry $7-13$ ). This is in contrast to reported in-solution depolymerization of PET by $\mathrm{HiC}$, which is efficient only above $60^{\circ} \mathrm{C}$ and with low crystallinity PET (Table S10).(21) Varying the liquid-to-solid ratio between 
0.5-2 $\mu \mathrm{L} \mathrm{mg}^{-1}$ (Fig. 2C, Table S2 entries 6-9) demonstrated that HiC functions well even in drier conditions, allowing STY of $1.58 \mathrm{gTPA}^{-1} \mathrm{~h}^{-1}$. This is remarkable, considering that a liquid-to-solid ratio $\eta=0.5 \mu \mathrm{L} \mathrm{mg}^{-1}$ corresponds to only 2.5 -fold molar excess of water in the HiC-catalysed PET hydrolysis.

The HiC enzyme maintained a similar level of activity over a pH range of 6-9 (Fig. 2D, Table S1 entries 4, 15-17), regardless of the nature and concentration $(0.1-1 \mathrm{M})$ of the buffer (Table S1 entries 19,20$)$. Based on the poor water solubility of TPA $\left(0.015 \mathrm{mg} \mathrm{mL}^{-1}\right.$ at $\left.20^{\circ} \mathrm{C}\right)$, it is not anticipated to dissolve significantly in the buffer. Indeed, powder X-ray diffraction (PXRD) patterns recorded after 7 days of aging at $55^{\circ} \mathrm{C}$ (Fig. S4) show the presence of crystalline TPA, which is not expected to meaningfully affect the $\mathrm{pH}$ of the buffer, consistent with the tolerance of the reaction for a broad range of buffer concentrations. Reactions carried out without buffer (only water) gave a slightly lower TPA yield of $14 \pm 1 \%$ (Table S1 entry 18).

\section{Hydrolysis of post-consumer PET and other plastics}

Exploring the efficacy of our process on post-consumer PET (Table S3), we first investigated the hydrolysis of three powdered, transparent post-consumer PET bottles (30-35\% crystalline, transparent, green and light blue, see SI for particle size characterization). Ball milling (5 or 30 minutes) and aging (7 days) of these PET powders with $0.6 \mathrm{wt} \%$ of $\mathrm{HiC}$, at $\eta=1.5 \mu \mathrm{L} \mathrm{mg}{ }^{-1}$ of 0.1 $M$ sodium phosphate buffer ( $\mathrm{pH} 7.3$ ) gave $16 \pm 2 \%$ (transparent), $13.0 \pm 0.4 \%$ (green) and $14.4 \pm$ $0.7 \%$ (light blue) yield of TPA. Notably, comparable TPA yield (15 $\pm 4 \%$ ) was reached by milling intact transparent PET bottle pieces with the enzyme before aging, suggesting that premicronisation of the sample could be avoided. An equal yield of TPA was achieved when the bottlesourced PET was pre-milled with $10 \mathrm{wt} \%$ of polypropylene cap pieces. Similarly, a powdered black post-consumer PET container, labelled as containing $80 \%$ recycled content, yielded $15 \pm 1 \%$ TPA under similar conditions. The addition of microcrystalline cellulose or polystyrene to the reaction mixture did not affect PET depolymerization by HiC either (Table S4). These results show that PET hydrolysis in moist-solid environment can proceed even in the presence of common post-consumer plastics impurities.

Lastly, we explored the potential of our process for depolymerizing other plastics. We found that modest depolymerization of polybutylene terephthalate (PBT) and polycarbonate (PC) was achieved even with mechanoenzymatic reaction conditions optimized for PET, consistently outperforming in-solution reactions (Table S5).

\section{Acceleration of PET hydrolysis by reactive aging (RAging)}

Similar to enzymatic kinetics in solution, previous reports on the mechanoenzymatic breakdown of the biopolymers cellulose,(22-24) xylan(25), and chitin(26) demonstrated that enzyme kinetics under aging conditions are hyperbolic. Such a relationship is typically explained by gradual substrate depletion and/or progressive enzyme denaturation (27). These reactions can be accelerated by periodically repeating the mechanical mixing just before the yield reaches a plateau, in a process termed reactive aging (RAging).(23)

Kinetic analysis of the HiC-catalyzed PET depolymerization (after 5 minutes of milling) revealed an initial rate of $10.4 \mathrm{mMTPA}^{-1}$, that decreased rapidly after 24 hours (Fig. 1C, also see $\mathrm{SI})$. Given this information, we elected to use RAging cycles consisting of 5 minutes ball milling at $30 \mathrm{~Hz}$ and 24 hours aging at $55^{\circ} \mathrm{C}$. This led to a $21 \pm 2 \%$ yield of TPA in only 3 days (Fig. $3 \mathrm{~A}$, Table S6 entry 1), compared to 7 days needed to achieve the same yield if milling is performed only once. The RAging reaction was also successfully scaled up 3-fold, using the same $15 \mathrm{~mL}$ milling jars, without any decrease in conversion (Table S6 entry 2). While RAging was clearly advantageous to speed up depolymerization, the reactions typically converged at $20-25 \%$ yield (Fig. 3A).

\section{Mechanistic considerations}

We found that this plateau at $20-25 \%$ yield was not broken by increasing the enzyme loading (Fig. $3 \mathrm{~A}$, Table S6 entry 3), and was not the result of product inhibition (Table S7 entries 1-3), structural 
changes to the PET material during the reaction (Figs. S2, S4, S5), or mechanical denaturation of the enzyme, as active $\mathrm{HiC}$ was easily extracted into buffer from the reaction mixture after 5 minutes of milling (Table S8, entry 3). Similarly, $\mathrm{HiC}$ retained activity even after 7 days of aging at $55^{\circ} \mathrm{C}$ in a reaction with the poorer substrate polybutylene terephthalate (Table S8, entry 7 ), yet no active enzyme could be extracted from PET reaction mixtures at or near the plateau (Table S8, entries 4 and 5). We observed that after separation of the hydrolysis products (see SI section TPA isolation), $\mathrm{HiC}$ remained on the PET surface (Fig. S14). This surface-bound $\mathrm{HiC}$ was found to exhibit only about $4 \%$ of its original activity (Table S8 entries 8 and 9 ), allowing us to conclude that after 3 days of reaction, most of the protein is denatured and adsorbed on PET. Together, these results imply that adsorption to the PET surface likely arises after activity-induced denaturation of the enzyme (such denaturation was also observed for example with P450 enzymes, which remain stable longer during storage, than when catalyzing a reaction at the same temperature(28)). This may, in principle, be overcome by switching to a different enzyme or by protein engineering.

As the reaction progressed, the PET crystallinity (measured by differential scanning calorimetry or DSC; Fig. S2) did not vary significantly, indicating that both crystalline and amorphous PET regions were hydrolysed to the same extent. This is unexpected and in stark contrast with the previously reported activity (in buffer) of PETase, leaf-branch compost cutinase and even $\mathrm{HiC}$, which preferentially depolymerized amorphous PET.(21, 29-34) Pre-milling of amorphous thin film PET (13\% crystallinity) increased the crystallinity to 33\% (Fig. S3, Table S3 entry 5),(35) which demonstrated that no amorphization of PET takes place during milling (this however prevented us from acquiring data for low-crystallinity PET powders). The viscosity average molecular weight of PET did not change drastically as the reaction progressed, suggesting that $\mathrm{HiC}$ hydrolyses PET polymers mainly from the end of the chains (see SI). Finally, scanning electron microscopy (SEM) images revealed no significant pitting of the remaining PET (Figs. 3B, 3C), implying that depolymerization occurs evenly across the surface.

\section{Maximizing the yield of TPA from PET}

Given the above results, it was envisaged that PET depolymerization might be improved if the enzyme was added in batches, i.e. by replenishing the denatured enzyme once the reaction reaches a plateau, i.e. after 3 days. In order to keep the liquid-to-solid ratio constant at $\eta=1.5 \mu \mathrm{L}$ $\mathrm{mg}^{-1}$, the remaining PET was washed and dried before adding fresh enzyme and buffer. After the initial 3-cycle RAging process which reached a TPA yield of $17.0 \pm 0.6 \%$, all following rounds proceeded with an approximate $10 \%$ additional yield of TPA from the remaining PET (Fig. 4, gray), allowing us to reach an overall $49 \pm 2 \%$ yield of TPA from $900 \mathrm{mg}$ of PET over 7 rounds, using a total of 3 wt\% of enzyme (Fig. 4, Table S9 entry 1 ).

The PET particles remaining after this process are small and exhibit increased surface roughness (Fig. 4 bottom right). Enzyme retention on the hydrolysed PET surface was confirmed again by energy dispersive X-ray spectroscopic analysis (EDS), showing the presence of nitrogen on the reacted PET particles after washing (Figs. S9, S10), but not on unused PET (Fig. S8). The reason for the lower PET conversion past the first round is unknown (Fig. 4, gray), but may be caused by several parameters (polydispersity, enzyme adsorption, reaction kinetics, additives in the plastic, etc.). Addition of denatured non-catalytic protein on the surface of PET (0.6 wt\% of bovine serum albumin) prior to the reaction did not affect the activity of HiC (Fig. S15), implying that this amount of denatured protein is unlikely to physically limit the active surface area of PET.

For comparison, control multi-round reactions were also performed under conventional dilute conditions $\left(\eta=50 \mu \mathrm{L} \mathrm{mg}^{-1}\right.$ i.e. $\left.20 \mathrm{mg} \mathrm{ml}^{-1}\right)$ with gentle shaking. The reaction conditions were otherwise identical to the above multi-round reactions in moist-solid mixtures (e.g. same enzymeto-substrate ratio and with isolation of the remaining dried PET before adding fresh enzyme and buffer). Overall yields of $21 \pm 3 \%$ MHET and $10 \pm 2 \%$ of TPA were obtained after 7 rounds (Table S9 entry 3 ), indicating that reactions in moist-solid mixtures afford a better yield and proceed with significantly higher selectivity for TPA ( $49 \pm 2 \%$ TPA and $\leq 4 \%$ MHET)).

We also performed multi-round reactions in moist-solid mixtures without product removal, which yielded $38 \pm 1 \%$ TPA after 7 rounds (Table S9 entry 2). This suggests that washing the substrate may increase the PET surface accessibility to the enzyme. Comparison to an equivalent 
reaction performed under conventional aqueous conditions ( $\eta=50 \mu \mathrm{L} \mathrm{mg}^{-1}$, i.e. $20 \mathrm{mg} \mathrm{ml}^{-1}$ ) shows that batch addition of fresh enzyme to suspended PET, without product removal, yielded only 14.8 $\pm 0.3 \%$ TPA (Table S9 entry 4). The consistently higher yields achieved in moist-solid mixtures may stem from slower build-up of enzyme and product molecules on the PET surface.

The TPA produced by mechanoenzymatic hydrolysis of PET is readily isolated from the reaction mixture by washing with methanol, or more conveniently with aqueous $\mathrm{Na}_{2} \mathrm{CO}_{3}$, to obtain a water-soluble salt from which TPA can be precipitated by acidification (see $\mathrm{SI}$ ). Product analysis by PXRD (Fig. S4), FTIR (Figs. S6, S7), and ${ }^{1} \mathrm{H}$ NMR (Figs. S11-S13) reveals that the isolated TPA is crystalline and $>95 \%$ pure, with the only minor product detected being MHET ( $\leq 4 \mathrm{wt} \%)$.

\section{Conclusion}

Using enzymes in moist-solid reaction mixtures rather than in traditional dilute solutions or slurries is an emergent strategy(36) that has proven beneficial for the breakdown of recalcitrant biopolymers such as cellulose, $(22,23)$ xylan, $(25)$ and chitin(26). Such reaction conditions provide a milieu that is closer to the natural setting of enzymes like $\mathrm{HiC}$, which are secreted in the environment by microorganisms. We demonstrate herein that, in contrast to enzymes used in dilute buffer, this approach enables the direct enzymatic depolymerization of high crystallinity PET, including post-consumer packaging, and even mixed plastics, while avoiding the energy-expensive melt-amorphization step, currently considered necessary for efficient enzymatic PET depolymerization(13). In addition to avoiding harsh chemicals, elevated temperatures, and high pressures, this strategy also minimizes the total reaction volume, thereby avoiding solubility issues, greatly facilitating handling and mixing, and curtailing waste. Achieving $>95 \%$ pure TPA in nearly $50 \%$ yield with $3 \mathrm{wt} \%$ enzyme from highly crystalline PET our methodology reaches 13 -fold higher STY and 15-fold higher yield per gram of enzyme compared to previous studies with high crystallinity PET. Overall, our method enables the recycling of PET plastics under milder, more sustainable conditions. Although validated here with the commercial wild type HiC enzyme, it should be especially promising for engineered proteins.

\section{Materials and Methods}

This section includes a general description of the enzymatic reaction conditions used with PET substrates. Full details on Materials and Methods, including equipment used, are provided in the Supporting Information (SI), which also includes Figures S1-S15: Characterization of the commercial Humicola insolens cutinase (HiC) enzyme (S1), DSC heating scans (S2-S3), PXRD patterns of PET, the reaction mixtures and the products (S4), FTIR spectra (S5-S7), SEM images with EDX analysis (S8-S10), ${ }^{1} \mathrm{H}$ NMR spectra of isolated products (S11-S13), Coomassie Blue G250 protein staining tests (S14) and denatured BSA test (S15), as well as Tables S1-S9 which summarize all results, and Table $\mathbf{S 1 0}$ which combines the space-time yields of TPA production by enzymatic hydrolysis of PET in previous literature.

\section{General method for reactions treated with milling once + aging}

In a typical reaction, PET (300 mg, $1.58 \mathrm{mmol})$ was weighed into a $15 \mathrm{~mL}$ PTFE or stainless-steel jar, charged either with $\mathrm{ZrO}_{2}$ or stainless-steel ball(s), to which the commercial enzyme preparation (300 $\mu \mathrm{L}, 1.95 \mathrm{mg}$ protein) and buffer $(150 \mu \mathrm{L})$ were added. The liquid-to-solid ratio is defined as $\eta$ and expressed in $\mu \mathrm{L} \mathrm{mg}^{-1}$. The jar was mounted on a ball mill, before milling at a frequency of 30 $\mathrm{Hz}$ for 5 minutes. The resulting solids were aliquoted into three parts, $10-50 \mathrm{mg}$ each, for analysis of the reaction products by HPLC at three time points: 1) after milling, 2) after milling followed by 3 days of aging at $55^{\circ} \mathrm{C}$, and 3) after milling followed by 7 days of aging at $55^{\circ} \mathrm{C}$. Separate aliquots were prepared for PXRD and NMR analysis of the reaction mixture. Specific reaction conditions and variations tested, together with corresponding hydrolysis yields are compiled into Table S1 and Table S3. Hydrolysis yield of similar reactions carried out with lyophilized enzyme are further described in the SI (Methods and Table S2). Similarly, reaction conditions and results from studying the influence of non-participating polymers (Table S4) and product inhibition (Table S7) on the 
mechanoenzymatic degradation of PET are included, and details on the depolymerizations of PBT and PC polymers (Table S5).

\section{General method for reactions using RAging conditions}

In a typical RAging reaction, PET ( $300 \mathrm{mg}, 1.58 \mathrm{mmol}$ ) was weighed into a $15 \mathrm{~mL}$ unsleeved PTFE jar, charged with a single $10 \mathrm{~mm} \mathrm{ZrO}_{2}$ ball $(3.5 \mathrm{~g})$, to which the commercial enzyme preparation $(300 \mu \mathrm{L}, 1.95 \mathrm{mg}$ protein) and buffer $(150 \mu \mathrm{L})$ were added. A cycle of RAging typically consisted of ball milling at $30 \mathrm{~Hz}$ for 5 minutes followed by aging at $55^{\circ} \mathrm{C}$ for 24 hours. These cycles were repeated up to 7 times, with an aliquot $(10-20 \mathrm{mg})$ collected at each cycle for analysis of the reaction products by HPLC. Sealing tape proved to be necessary to ensure that the jars remain closed during the aging step. Specific reaction conditions and variations tested, together with corresponding hydrolysis yields are compiled into Table S6.

\section{TPA isolation}

The TPA in the solid reaction mixture was reacted with 1.2 eq of sodium carbonate (added as 0.15 $\mathrm{M}$ aqueous solution), to yield a water-soluble sodium terephthalate salt that was easily extracted from the remaining PET with $3 \times 3 \mathrm{~mL}$ MilliQ water. The combined aqueous solutions of sodium sodium terephthalate were filtered through cotton and acidified with 2 molar equivalents of $\mathrm{HCl}$ to $\mathrm{pH} 1-2$, resulting in the precipitation of TPA. The TPA was isolated by centrifugation $(5000 \times g)$ and removal of the acidic supernatant. The product was washed with water until washings were neutral, and lyophilized to yield a white powder. According to ${ }^{1} \mathrm{H}$ NMR analysis (Fig. S13) the isolated product contains mainly TPA (96 wt\%), with the minor impurity from MHET (4 wt\%). The isolated reaction products were also analyzed by PXRD (Fig. S4) and FTIR (Fig. S6).

\section{Multi-round RAging experiments}

PET (900 mg, $4.74 \mathrm{mmol}$ ) was weighed into a $15 \mathrm{~mL}$ unsleeved PTFE jar, charged with a single $10 \mathrm{~mm} \mathrm{ZrO} 2$ ball $(3.5 \mathrm{~g})$, to which the buffer $(450 \mu \mathrm{L})$ and the commercial enzyme preparation (900 $\mu \mathrm{L}, 5.85 \mathrm{mg}$ protein) were added. Control reactions were carried out with the flow-through $(900 \mu \mathrm{L})$ from the commercial enzyme solution, obtained by using a $10 \mathrm{kDa}$ MWCO centrifugal concentrator. The multi-round experiment consisted of seven rounds, each with three RAging cycles (5 minutes of milling at $30 \mathrm{~Hz}$, followed by aging at $55^{\circ} \mathrm{C}$ for 24 hours) - totaling 21 RAging cycles. After every round, hydrolysis products were removed by transferring the solid paste to a $15 \mathrm{~mL}$ conical tube, before addition of $0.3 \mathrm{M} \mathrm{Na}_{2} \mathrm{CO}_{3}$, vortexing and centrifugation at $10,000 \times g$ for $5 \mathrm{~min}$. The supernatant (containing TPA) was kept aside and the remaining PET residue was washed with MilliQ water $(3 \times 3 \mathrm{~mL})$, until neutral $\mathrm{pH}$ was reached, frozen in liquid nitrogen and lyophilized before being used a the substrate in the next round: weighed into a $15 \mathrm{~mL}$ unsleeved PTFE jar, charged with a single $10 \mathrm{~mm} \mathrm{ZrO}$ ball $(3.5 \mathrm{~g})$, and the corresponding amount of enzyme preparation $(0.65 \%$ Wrotein/Wsolid) and buffer to maintain an $\eta$ of $1.5 \mu \mathrm{L} \mathrm{mg}^{-1}$. An aliquot (10-20 mg) was taken for HPLC analysis at the start and end of each round. The results are displayed on Fig. 5 and in Table S6. The remaining PET was characterized by SEM (with EDX) after the first and seventh rounds, as seen in Figs. S9 and S10, and compared with non-hydrolysed PET (Fig. S8). A multi-round RAging experiment, without the isolation of products and with the enzyme added as a lyophilized powder was also carried out, which is described further in the SI.

\section{Acknowledgments}

We thank NSERC (RGPIN-2017-06467, SMFSU 507347-17), FRQNT (PR 254169), the McGill Sustainability Systems Initiative, and the Centre in Green Chemistry and Catalysis for funding (FRQNT-2020-RS4-265155-CCVC).

\section{References}

1. PlasticsEurope, Plastics-the facts 2019. An analysis of European plastics production, demand and waste data. (2019). 
(C) 2021 National Academy of Sciences.

2. R. Geyer, J. R. Jambeck, K. L. Law, Production, use, and fate of all plastics ever made. Sci. Adv. 3, 25-29 (2017).

3. IHS Markit, "PET polymer: chemical economics handbook." (2019).

4. J. H. Lee, K. S. Lim, W. G. Hahm, S. H. Kim, Properties of recycled and virgin poly(ethylene terephthalate) blend fibers. J. Appl. Polym. Sci. 128, 1250-1256 (2013).

5. K. Ragaert, L. Delva, K. Van Geem, Mechanical and chemical recycling of solid plastic waste. Waste Manag. 69, 24-58 (2017).

6. I. Vollmer, et al., Beyond Mechanical Recycling: Giving New Life to Plastic Waste. Angew. Chem. Int. Ed. 59, 15402-15423 (2020).

7. T. Yoshioka, T. Sato, A. Okuwaki, Hydrolysis of waste PET by sulfuric acid at $150^{\circ} \mathrm{C}$ for a chemical recycling. J. Appl. Polym. Sci 52, 1353-1355 (1994).

8. D. Paszun, T. Spychaj, Chemical Recycling of Poly(ethylene terephthalate). Ind. Eng. Chem. Res. 36, 1373-1383 (1997).

9. T. Yoshioka, N. Okayama, A. Okuwaki, Kinetics of Hydrolysis of PET Powder in Nitric Acid by a Modified Shrinking-Core Model. Ind. Eng. Chem. Res. 37, 336-340 (1998).

10. G. P. Karayannidis, A. P. Chatziavgoustis, D. S. Achilias, Poly(ethylene terephthalate) recycling and recovery of pure terephthalic acid by alkaline hydrolysis. Adv. Polym. Technol. 21, 250-259 (2002).

11. D. Carta, G. Cao, C. D'Angeli, Chemical recycling of poly(ethylene terephthalate) (pet) by hydrolysis and glycolysis. Environ. Sci. Pollut. Res. 10, 390-394 (2003).

12. B. Shojaei, M. Abtahi, M. Najafi, Chemical recycling of PET: A stepping-stone toward sustainability. Polym. Adv. Technol. 31, 2912-2938 (2020).

13. V. Tournier, et al., An engineered PET depolymerase to break down and recycle plastic bottles. Nature 580, 216-219 (2020).

14. S. Kaabel, T. Friščić, K. Auclair, Mechanoenzymatic Transformations in the Absence of Bulk Water: A More Natural Way of Using Enzymes. ChemBioChem 21, 742-758 (2020).

15. T. Friščić, S. L. Childs, S. A. A. Rizvi, W. Jones, The role of solvent in mechanochemical and sonochemical cocrystal formation: a solubility-based approach for predicting cocrystallisation outcome. CrystEngComm 11, 418-426 (2009).

16. D. Hasa, E. Miniussi, W. Jones, Mechanochemical Synthesis of Multicomponent Crystals: One Liquid for One Polymorph? A Myth to Dispel. Cryst. Growth Des. 16, 4582-4588 (2016).

17. D. Hasa, W. Jones, Screening for new pharmaceutical solid forms using mechanochemistry: A practical guide. Adv. Drug Deliver. Rev. 117, 147-161 (2017).

18. M. J. Cliffe, C. Mottillo, R. S. Stein, D.-K. Bučar, T. Friščić, Accelerated aging: a low energy, solvent-free alternative to solvothermal and mechanochemical synthesis of metalorganic materials. Chem. Sci. 3, 2495-2500 (2012).

19. C. Mottillo, et al., Mineral neogenesis as an inspiration for mild, solvent-free synthesis of bulk microporous metal-organic frameworks from metal ( $\mathrm{Zn}, \mathrm{Co})$ oxides. Green Chem. 15, 2121-2131 (2013). 
(C) 2021 National Academy of Sciences.

20. C. Mottillo, T. Friščić, Advances in Solid-State Transformations of Coordination Bonds: From the Ball Mill to the Aging Chamber. Molecules 22, 144 (2017).

21. Å. M. Ronkvist, W. Xie, W. Lu, R. A. Gross, Cutinase-Catalyzed Hydrolysis of Poly(ethylene terephthalate). Macromolecules 42, 5128-5138 (2009).

22. F. Hammerer, et al., Rapid mechanoenzymatic saccharification of lignocellulosic biomass without bulk water or chemical pre-treatment. Green Chem. 22, 3877-3884 (2020).

23. F. Hammerer, et al., Solvent-Free Enzyme Activity: Quick, High-Yielding Mechanoenzymatic Hydrolysis of Cellulose into Glucose. Angew. Chem. Int. Ed. 57, 2621-2624 (2018).

24. F. Hammerer, S. Ostadjoo, T. Friščić, K. Auclair, Towards Controlling the Reactivity of Enzymes in Mechanochemistry: Inert Surfaces Protect $\beta$-Glucosidase Activity During Ball Milling. ChemSusChem 13, 106-110 (2020).

25. Ostadjoo, et al., Efficient Enzymatic Hydrolysis of Biomass Hemicellulose in the Absence of Bulk Water. Molecules 24, 4206 (2019).

26. J. P. D. Therien, F. Hammerer, T. Friščić, K. Auclair, Mechanoenzymatic Breakdown of Chitinous Material to N -Acetylglucosamine: The Benefits of a Solventless Environment. ChemSusChem 12, 3481-3490 (2019).

27. R. G. Duggleby, "Analysis of enzyme progress curves by nonlinear regression" in Methods in Enzymology, (1995), pp. 61-90.

28. A. Chefson, J. Zhao, K. Auclair, Replacement of Natural Cofactors by Selected Hydrogen Peroxide Donors or Organic Peroxides Results in Improved Activity for CYP3A4 and CYP2D6. ChemBioChem 7, 916-919 (2006).

29. H. P. Austin, et al., Characterization and engineering of a plastic-degrading aromatic polyesterase. Proc. Natl. Acad. Sci. U.S.A. 115, E4350-E4357 (2018).

30. R. Wei, et al., Biocatalytic Degradation Efficiency of Postconsumer Polyethylene Terephthalate Packaging Determined by Their Polymer Microstructures. Adv. Sci. 6, 1900491 (2019).

31. I. Donelli, et al., Enzymatic surface modification and functionalization of PET: A water contact angle, FTIR, and fluorescence spectroscopy study. Biotechnol. Bioeng. 103, 845856 (2009).

32. K. Herzog, R.-J. Müller, W.-D. Deckwer, Mechanism and kinetics of the enzymatic hydrolysis of polyester nanoparticles by lipases. Polym. Degrad. Stab. 91, 2486-2498 (2006).

33. T. Brueckner, A. Eberl, S. Heumann, M. Rabe, G. M. Guebitz, Enzymatic and chemical hydrolysis of poly(ethylene terephthalate) fabrics. J. Polym. Sci. A1 46, 6435-6443 (2008).

34. I. Donelli, G. Freddi, V. A. Nierstrasz, P. Taddei, Surface structure and properties of poly(ethylene terephthalate) hydrolyzed by alkali and cutinase. Polym. Degrad. Stab. 95, 1542-1550 (2010).

35. A. Misra, R. S. Stein, Stress-induced crystallization of poly(ethylene terephthalate). J. Polym. Sci. Polym. Phys. Ed. 17, 235-257 (1979).

36. K. Auclair, T. Friščić, S. Kaabel, J. P. D. Therien, Mechanoenzymatic degradation of 
(C) 2021 National Academy of Sciences.

polymers. PCT filed Oct 27, 2020, CA2020051438. 


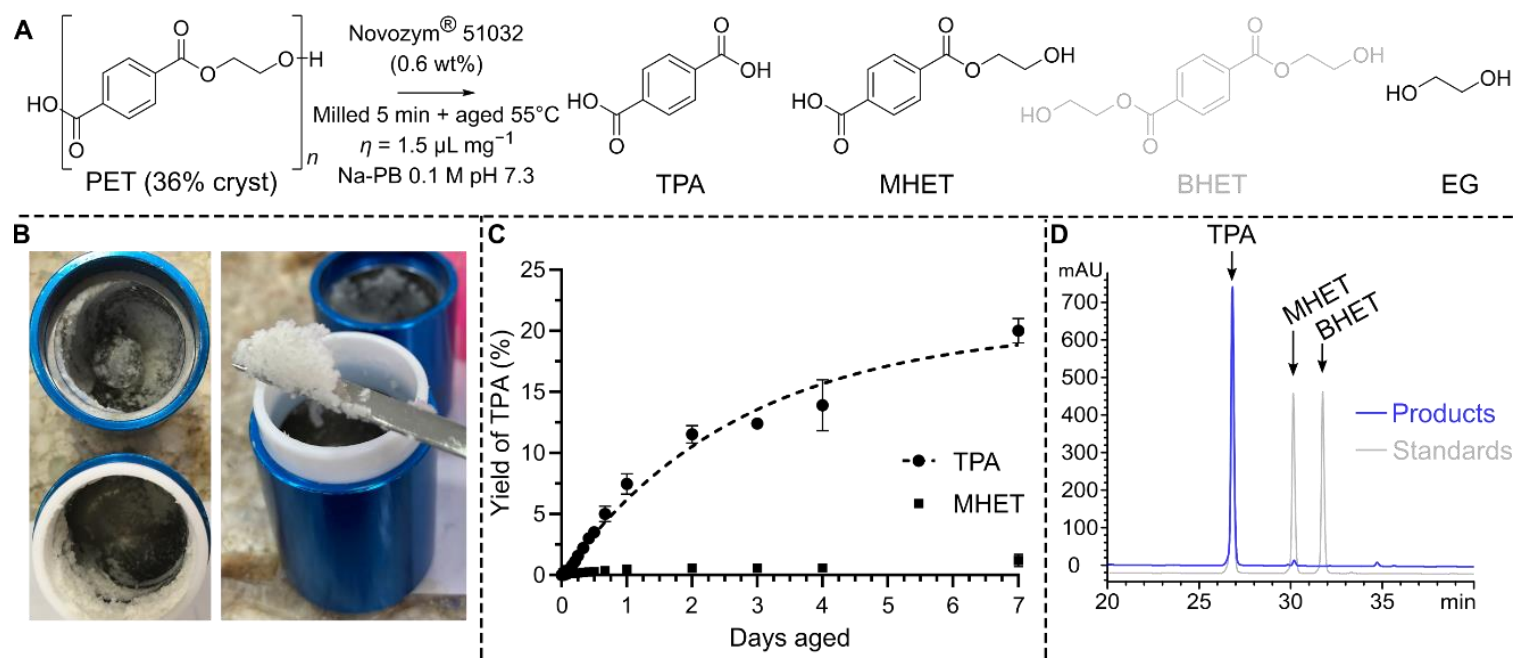

Figure 1. A) General reaction scheme of the mechanoenzymatic hydrolysis of PET using HiC (Novozym® 51032), showing the possible hydrolysis products terephthalic acid (TPA), mono(2hydroxyethyl) terephthalate (MHET), bis(2-hydroxyethyl) terephthalate (BHET) and ethylene glycol (EG). BHET (in gray) was not detected. B) Photos of the moist-solid reaction mixture after 5 minutes of milling. C) The kinetics of $\mathrm{HiC}$ during 7 days of aging at $55^{\circ} \mathrm{C}$ on PET powder (36\% crystallinity), given as yield of TPA (\% of theoretical), with a calculated initial rate of $10.4 \mathrm{mMTPA} \mathrm{h}^{-1}$. All experiments were conducted in triplicate, error bars show the standard deviation. D) Chromatograms of the reaction products (blue) compared to traces of standard samples of TPA, MHET and BHET (gray) monitored at $240 \mathrm{~nm}$.
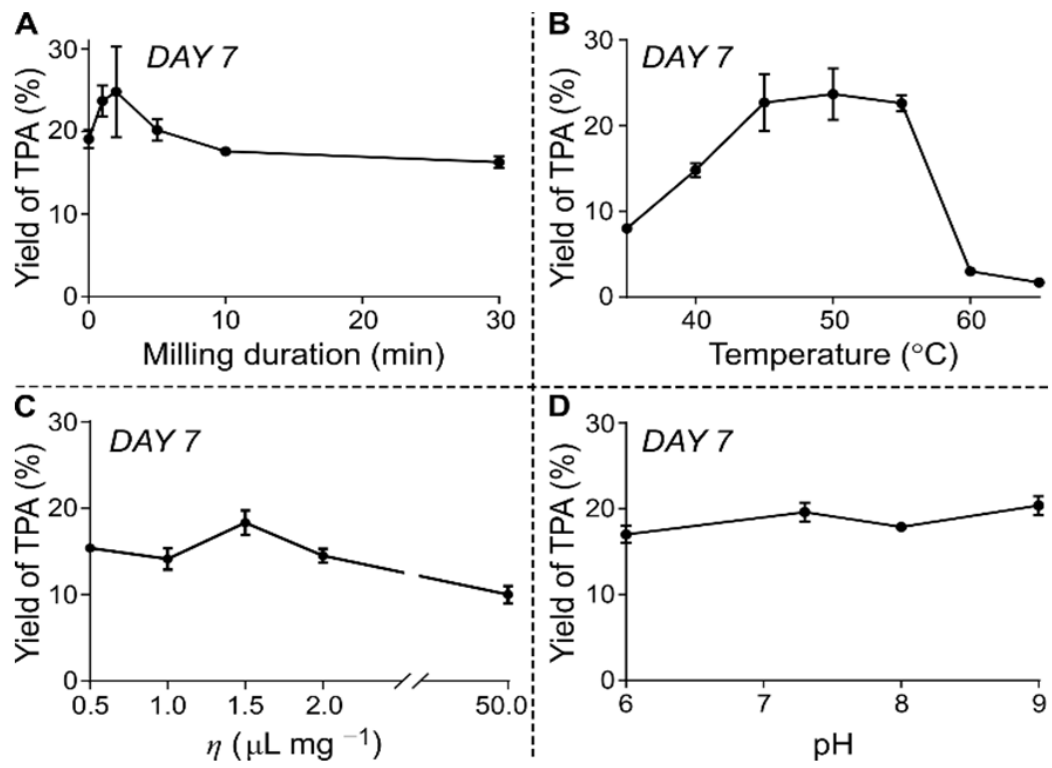

Figure 2. Reaction outcome (day 7) depending on the duration of the initial milling where 0 min is manual mixing (A), at different aging temperatures (B), at different liquid-to-solid ratios (C) and at different buffer $\mathrm{pH}(\mathrm{D})$. All experiments were conducted in triplicate, error bars show the standard deviation. 

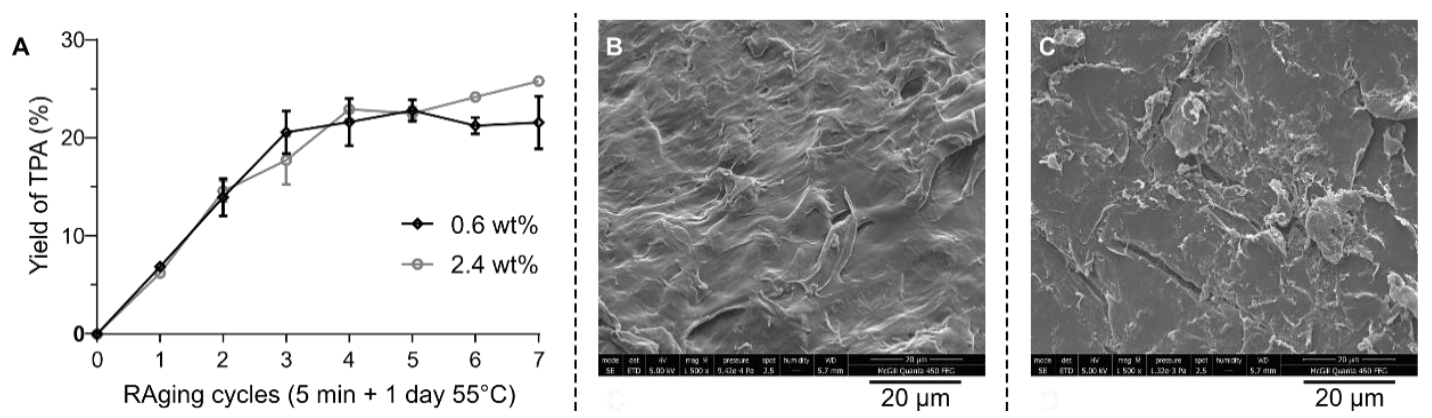

Figure 3. (A) Formation of TPA (\%) during RAging reactions at 0.6 wt $\%$ (black) or 2.4 wt\% (gray) $\mathrm{HiC}$ loading. All experiments were conducted in triplicate, error bars show the standard deviation. SEM micrographs of the PET particles before (B) and after (C) the HiC-catalysed mechanoenzymatic reaction. Particles shown after the reaction were cleaned of the hydrolysis products by washing with methanol (see SI).

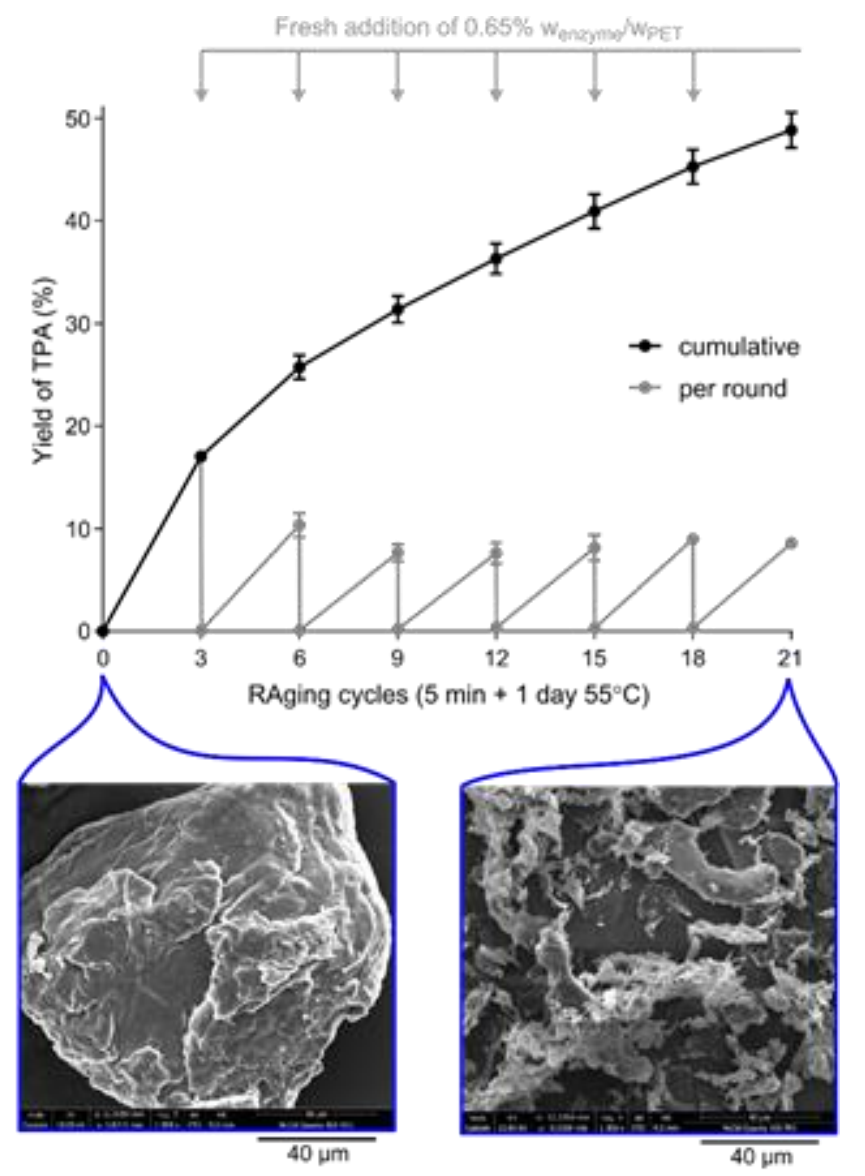

Figure 4. Per-round (gray) and cumulative yields (black) of TPA in the multi-round RAging experiments. All experiments were conducted in triplicate, error bars show the standard deviation. More enzyme $(0.65 \mathrm{wt} \%)$ and enough buffer to ensure a constant liquid-to-solid ratio were added after every round of RAging ( 3 cycles), and the obtained TPA was extracted. The reaction continued 
(C) 2021 National Academy of Sciences.

with the remaining washed and dried PET. SEM micrographs are shown (bottom) for the isolated and washed PET substrate both at the start of the reaction and for the remaining PET after the multi-round experiment. 